\title{
Review: antidepressants increase remission and clinical improvement in bulimia nervosa
}

\author{
Bacaltchuk J, Hay P.Antidepressants versus placebo for people with bulimia nervosa. Cochrane Database Syst Rev \\ 2001;(4):CD003391 (latest version 19 Aug 2001).
}

\section{QUESTION: In patients with bulimia nervosa, are antidepressants effective for increasing remission and clinical improvement?}

\section{Data sources}

Studies were identified by searching 7 databases, Clinical Evidence, and reference lists; by hand searching the International Journal of Eating Disorders; and by contacting authors and pharmaceutical companies.

\section{Study selection}

Studies were selected if they were randomised controlled trials (RCTs) that compared any antidepressant with placebo for $\geqslant 4$ weeks in patients with bulimia nervosa. RCTs were excluded if patients had binge eating or purging type anorexia nervosa or binge eating disorder.

\section{Data extraction}

2 reviewers assessed the quality of RCTs and extracted data on patients, study characteristics, drug regimens, and outcomes (including remission $[100 \%$ reduction in binge or purge episodes], clinical improvement [ $\geqslant 50 \%$ reduction in binge or purge episodes], and dropouts).

\section{Main results}

16 RCTs (1300 patients) met the selection criteria. Any antidepressant was better than placebo for increasing remission at a mean follow up of 8 weeks ( 8 RCTs) and clinical improvement at a mean follow up of 9 weeks ( 8 RCTs) (table). Groups did not differ for dropout rates (14 RCTs) (table).

\section{Conclusion}

In patients with bulimia nervosa, antidepressants are effective in the short term for increasing remission and clinical improvement rates.

Antidepressants v placebo for bulimia nervosa at 6-16 weeks*

Source of funding: not stated.

For correspondence: DrJ Bacaltchuk, Universidade Federal de São Paulo, São Paulo, Brazil. bacaltc@ibm.net

Abstract and commentary also appear in

Evidence-Based

Medicine.

\begin{tabular}{|c|c|c|c|c|}
\hline Outcomes & Antidepressant type & Weighted event rates & $\mathrm{RBI}(95 \% \mathrm{Cl})$ & NNT (Cl) \\
\hline \multirow[t]{4}{*}{ Remission } & All types & $20 \%$ v $7.9 \%$ & $105 \%$ (32 to 219$)$ & $9(6$ to 16$)$ \\
\hline & TCAs & $14 \%$ v $9.1 \%$ & $136 \%(-4$ to 476$)$ & Not significant \\
\hline & MAOIs & $25 \%$ v $6.3 \%$ & $229 \%(-22$ to 1289$)$ & Not significant \\
\hline & Other antidepressants & $15 \% \vee 0 \%$ & $664 \%(-1$ to 5773$)$ & 7 (4 to 27$)$ \\
\hline \multirow[t]{5}{*}{ Clinical improvement } & All types & $64 \% v 33 \%$ & $84 \%$ (38 to 145$)$ & 4 (3 to 5$)$ \\
\hline & TCAs & $77 \% \vee 17 \%$ & $294 \%$ (59 to 872 ) & $2(2$ to 3$)$ \\
\hline & SSRIs & $58 \% \vee 38 \%$ & $51 \%(26$ to 81$)$ & 5 (4 to 9$)$ \\
\hline & Other antidepressants & $44 \%$ v $8.2 \%$ & $321 \%$ (74 to 919$)$ & $3(3$ to 5$)$ \\
\hline & & & $\operatorname{RRR}(\mathrm{Cl})$ & NNT \\
\hline \multirow[t]{6}{*}{ Dropouts } & SSRIs & $34 \% \vee 40 \%$ & $18 \%$ (1 to 32$)$ & Not significant \\
\hline & Other antidepressants & $30 \% \vee 32 \%$ & $6 \%(-83$ to 52$)$ & Not significant \\
\hline & & & $\mathrm{RRI}(\mathrm{Cl})$ & NNH (CI) \\
\hline & All types & $34 \% \vee 31 \%$ & $3 \%(-20$ to 32$)$ & Not significant \\
\hline & TCAs & $26 \% \vee 11 \%$ & $93 \%$ (15 to 225$)$ & Not significant \\
\hline & MAOIs & $34 \%$ v $29 \%$ & $20 \%(-33$ to 113$)$ & Not significant \\
\hline
\end{tabular}

*MAOIs=monoamine oxidase inhibitors; SSRIs=selective serotonin reuptake inhibitors; TCAs=tricyclic agents. Other abbreviations defined in glossary; RBI, RRI, NNT, $\mathrm{NNH}$, and $\mathrm{Cl}$ calculated from data in article.

\section{COMMENTARY}

The reviews by Bacaltchuk et al are laudable for the rigour of the data analyses, but they rightly generate more questions than answers. Firstly, Bacaltchuk and Hay have comprehensively reviewed 16 published RCTs of antidepressant treatments for bulimia nervosa (BN). Although modest effectiveness is shown, high dropout rates among patients limit the clinical application of these data, and the authors comment on the need for more studies of tolerability and cost effectiveness. The studies included were generally of short duration in young adult women who did not have any substantial psychiatric comorbidity. The results cannot therefore be generalised to the substantial minority of bulimic patients with comorbid "multi-impulsive" personality characteristics, ${ }^{1}$ to patients with substance misuse, or to adolescents.

Pharmacological treatment trials of $\mathrm{BN}$ are dominated by the reported reduction in bulimic symptoms, but clinicians and their patients are more interested in remission of symptoms. The emphasis of this review on remission is therefore of greater clinical application than the emphasis of its sources. The review discusses the limitations of outcome measures and is right to conclude that the use of antidepressants as sole therapy "does not seem sufficient to effectively treat these patients".

Secondly, Bacaltchuk et al review a scant number of studies comparing combined antidepressant medication and psychotherapy with each treatment alone. In clinical practice, cognitive behavioural therapy (CBT) is generally regarded as the treatment of choice for BN, which is limited by its availability, with antidepressant medication as an adjunct. This review supports that approach with restricted data from fairly small studies. However, the clinical risk associated with a pharmacological approach to BN seems to be a higher dropout rate than CBT, and again the results cannot necessarily be generalised beyond young adult women without substantial comorbidity.

The UK Department of Health's National Service Framework for Mental Health has stressed the importance of managing such eating disorders as BN in primary care, ${ }^{2}$ noting that "antidepressants can reduce purging and bingeing whether or not the person is also depressed". Although true in the short term, this would seem an optimistic reading of the literature. Prescription of antidepressants may appear to be the easiest route in a primary care setting. The clinical implication of the review by Bacaltchuk et al is that the easiest route may not be the most effective, cost effective, or acceptable for clinicians and their patients. 


\title{
Review: psychological treatment is as effective as antidepressants for bulimia nervosa, but a combination is best
}

\author{
Bacaltchuk J, Hay P, Trefiglio R. Antidepressants versus psychological treatments and their combination for bulimia \\ nervosa. Cochrane Database Syst Rev 2001;(4):CD003385 (latest version 13 Aug 2001).
}

QUESTIONS: In patients with bulimia nervosa (BN), are antidepressants as effective as psychological treatment (PT) for increasing remission and clinical improvement rates? Is a combination of antidepressants and PT better than each intervention alone?

\section{Data sources}

Studies were identified by searching Medline; EMBASE/ Excerpta Medica; LILACS; PsychLIT; SCISEARCH; the Cochrane Depression, Anxiety, and Neurosis Group Database of Trials; the Cochrane Controlled Trials Register; Clinical Evidence; and reference lists; by hand searching the International Journal of Eating Disorders and book chapters on $\mathrm{BN}$; and by contacting authors and pharmaceutical companies.

\section{Study selection}

Studies were selected if they were randomised controlled trials (RCTs) that compared antidepressants with PT in patients with BN. Studies were excluded if patients had binge eating or purging type anorexia nervosa or binge eating disorder.

\section{Data extraction}

2 reviewers assessed the quality of studies and extracted data on patients, study characteristics, interventions, and outcomes (including remission $[100 \%$ reduction in binge or purge episodes], clinical improvement $[\geqslant 50 \%$ reduction in binge or purge episodes], and dropouts).

\section{Main results}

5 RCTs (237 patients) compared antidepressants with PT. Groups did not differ significantly for remission (5 RCTs); only 1 RCT reported on clinical improvement. More dropouts occurred in the antidepressant group than in the PT group (4 RCTs) (table). 5 RCTs (247 patients) compared combination and single interventions.

Antidepressants $v$ combination: more patients in the combination group than in the antidepressant alone group had remission (4 RCTs) (table); only 1 RCT reported on clinical improvement. Groups did not differ for dropout rates (4 RCTs).
$P T v$ combination: more patients in the combination group than in the PT alone group had remission (6 RCTs); fewer patients in the PT alone group than in the combination group dropped out (6 RCTs) (table). Groups did not differ for clinical improvement (2 RCTs) (table).

\section{Conclusions}

In patients with bulimia nervosa, psychological treatment (PT) and antidepressants do not differ in remission rates but dropout rates are lower with PT. A combination of antidepressants and PT is best for increasing remission.
Source of funding: not stated

For correspondence: Dr J Bacaltchuk, Universidade Federal de São Paulo, São Paulo, Brazil.

bacaltc@ibm.net

Abstract and commentary also appear in Evidence-Based Medicine.

Antidepressants $(A D)$ v psychological treatment (PT) for bulimia nervosa*

\begin{tabular}{|c|c|c|c|c|}
\hline Outcomes & Comparisons & $\begin{array}{l}\text { Weighted event } \\
\text { rates }\end{array}$ & RBI $(95 \%$ Cl) & NNT (CI) \\
\hline \multirow[t]{4}{*}{ Remission } & PT $v$ AD & $41 \%$ v $20 \%$ & $63 \%(-14$ to 210$)$ & Not significant \\
\hline & $A D+P T v A D$ & $47 \%$ v $23 \%$ & $79 \%$ (11 to 188$)$ & 5 (3 to 21$)$ \\
\hline & $\mathrm{PT}+\mathrm{AD} v \mathrm{PT}$ & $50 \%$ v $36 \%$ & $30 \%$ (1 to 68$)$ & 8 (5 to 37$)$ \\
\hline & & & RBR (CI) & NNH \\
\hline \multirow[t]{2}{*}{$\begin{array}{l}\text { Clinical } \\
\text { improvement }\end{array}$} & $\mathrm{PT}+\mathrm{AD} v \mathrm{PT}$ & $46 \%$ v $52 \%$ & $8 \%(-70$ to 50$)$ & Not significant \\
\hline & & & RRR (CI) & NNT (CI) \\
\hline \multirow[t]{4}{*}{ Dropouts } & PT $v$ AD & $18 \%$ v $41 \%$ & $54 \%$ (9 to 76 ) & 5 (3 to 10$)$ \\
\hline & $A D+P T \vee A D$ & $35 \%$ v 41\% & $16 \%(-45$ to 51$)$ & Not significant \\
\hline & & & RRI (CI) & $\mathrm{NNH}(\mathrm{Cl})$ \\
\hline & $\mathrm{PT}+\mathrm{AD} v \mathrm{PT}$ & $26 \% \vee 16 \%$ & $74 \%$ (14 to 167$)$ & $10(6$ to 40$)$ \\
\hline
\end{tabular}

* RBR=relative benefit reduction. Other abbreviations defined in glossary; RBI, RBR, RRI, RRR, NNT, NNH, and $\mathrm{Cl}$ calculated from data in article. Follow up ranged from 5-24 weeks.

\section{COMMENTARY—continued}

However, in the busy world of primary care, the treatment of BN will continue to be driven by available resources. Where specialists with training in CBT for BN are available, then that route is generally preferred by the family doctor. But the Royal College of Psychiatrists, in collaboration with the Consumers' Association, has recently reported the dearth of specialist eating disorder services beyond southeast England. ${ }^{3}$ Thus in the more likely scenario of limited eating disorder services, use of antidepressant medication may seem more attractive. These 2 reviews agree with that approach and suggest that antidepressant medication will produce short term results. BN, however, is not a short term illness. Relapse prevention deserves greater scrutiny for patients with $\mathrm{BN}$ and anorexia nervosa, and longer term follow up studies should drive the next generation of treatment intervention studies.

Regarding treatment of $\mathrm{BN}$ in particular, a pressing need exists for longer term studies examining relapse rates, health economics, and comparisons of classes of antidepressants for treatment concordance.

1 Lacey JH. Eating disorders and obesity: a comprehensive handbook. New York: Guilford Press, 1995:361-8.

2 A national service framework for mental health modern standards and service models. London: Department of Health, 1999.

3 Eating disorders in the UK: policies for service development and training. London: Royal College of Psychiatrists, August 2001. http://www.rcpsych.ac.uk/publications/cr/ council/cr87.pdf 